\title{
Is Chronic Curcumin Supplementation Neuroprotective Against Ischemia for Antioxidant Activity, Neurological Deficit, or Neuronal Apoptosis in an Experimental Stroke Model?
}

\author{
Serdar ALTINAY ${ }^{1}$, Murat CABALAR², Cihan ISLER ${ }^{3}$, Funda YILDIRIM ${ }^{4}$, Duygu S. CELIK5, Oguzhan ZENGI ${ }^{6}$, \\ Abdurrahim TAS ${ }^{7}$, Ahmet GULCUBUK ${ }^{4}$ \\ ${ }^{1}$ Selcuk University, Medical Faculty, Department of Pathology, Konya, Turkey \\ ${ }^{2}$ Ministry of Health, Health Science University, Bakirkoy Dr. Sadi Konuk Training and Research Hospital, Department of Neurology, \\ Istanbul,Turkey \\ ${ }^{3}$ Istanbul University, Cerrahpaşa Medical Faculty, Department of Neurosurgery, Istanbul, Turkey \\ ${ }^{4}$ Istanbul University, Istanbul Veterinary Faculty, Department of Pathology, Istanbul, Turkey \\ ${ }^{5}$ Ministry of Health, Health Science University, Bagcilar, Training and Research Hospital, Experimental Research Center, Istanbul,Turkey \\ ${ }^{6}$ Ministry of Health, Health Science University, Bagcilar, Training and Research Hospital, Department of Biochemistry, Istanbul, Turkey \\ ${ }^{7}$ Ministry of Health, Health Science University, Bagcilar, Training and Research Hospital, Department of Neurology, Istanbul, Turkey
}

\section{ABSTRACT}

AIM: To investigate the neuroprotective effect of chronic curcumin supplementation on the rat forebrain prior to ischemia and reperfusion.

MATERIAL and METHODS: Forebrain ischemia was induced by bilateral common carotid artery occlusion for $1 / 2$ hour, followed by reperfusion for 72 hours. Older rats were divided into five groups: Group I received $300 \mathrm{mg} / \mathrm{kg}$ oral curcumin for 21 days before ischemia and $300 \mathrm{mg} / \mathrm{kg}$ intraperitoneal curcumin after ischemia; Group II received $300 \mathrm{mg} / \mathrm{kg}$ intraperitoneal curcumin after ischemia; Group III received $300 \mathrm{mg} / \mathrm{kg}$ oral curcumin for 21 days before ischemia; Group IV had only ischemia; Group V was the sham-operated group. The forebrain was rapidly dissected for biochemical parameter assessment and histopathological examination.

RESULTS: In forebrain tissue, enzyme activities of superoxide dismutase, glutathione peroxidase, and catalase were significantly higher in Group I than Groups II or III $(p<0.05)$ while xanthine dehydrogenase and malondialdehyde enzyme activities and concentrations of interleukin- 6 and TNF-alpha were significantly lower in Group I when compared to Groups II and III ( $<<0.05)$. A significant reduction in neurological score was observed after 24 and 72 hours in the curcumin-treated groups compared with the ischemic group. We also found a marked reduction in apoptotic index after 72 hours in the groups receiving curcumin. Significantly more TUNEL-positive cells were observed in the ischemic group compared to those treated with curcumin.

CONCLUSION: We demonstrated the neuroprotective effect of chronic curcumin supplement on biochemical parameters, neurological scores and apoptosis following ischemia and reperfusion injury in rats.

KEYWORDS: Curcumin, Ischemia, Stroke, Neuroprotective effect, Oxidative stress 


\section{INTRODUCTION}

$\Lambda$ mong stroke subtypes including ischemia, intracerebral haemorrhage, and subarachnoid haemorrhage, the ischemic type constitutes $88 \%$ of all strokes worldwide $(6,26)$. When considering the epidemiology of cerebrovascular disease, in Turkey and elsewhere, a dramatic increase in life expectancy has led to a larger elderly population. Cerebrovascular disease is the second most frequent cause of death after cardiovascular disease in people over sixtyfive years of age and is the primary cause of disability and workforce loss $(6,8,26)$. In the United States, the incidence of first-time stroke is more than 700,000 per year, and $20 \%$ of patients die within one year after stroke onset (8).

There have been many major developments from molecular, genetic, clinical, and biochemical perspectives in ischemic stroke research in recent years, and many biochemical and immunological reactions have been explored in association with reduced cerebral blood flow. Several studies have revealed that ischemia causes excessive generation of free radicals while the antioxidant defence is impaired, increasing neuronal vulnerability and brain damage (11).

During ischemia and reperfusion, changes in mitochondrial oxidative phosphorylation, reduced ATP levels, increased intracellular $\mathrm{Ca}^{2+}$ concentrations, and protease and phosphatase activation lead to alterations in the cytoskeleton and membrane phospholipids and high levels of free oxygen radicals. It is reported that free oxygen radicals play a major role in the physiopathology of ischemia and reperfusion damage. After ischemia, much larger amounts of free radicals are produced following tissue re-oxygenation in the reperfusion period, and these species cause further damage by causing lipid peroxidation $(2,12)$.

An important member of the Zingiberaceae family, commonly known as "turmeric" or "Indian saffron," curcumin is registered as "Curcuma longa rhizome" by the World Health Organization. Curcuma longa $L$. is a yellow-flowered, large-leaved, perennial herbaceous plant that is native to South Asia and is widely used in medical treatment (4).

Curcumin is metabolised rapidly and efficiently, severely decreasing parent compound availability in the biophase. In an early rat study, a dietary dose $(1 \mathrm{~g} / \mathrm{kg})$ resulted in about $75 \%$ detection in faeces. Intravenous and intraperitoneal curcumin administration to rats resulted in large quantities of curcumin and metabolites in bile. Orally administered curcumin reportedly passes into the bloodstream and forms glucuronide and glucuronide sulphate conjugates. Therefore, the physiological effect must come from curcumin in these forms (19).

Ayurvedic medicine is the branch of medicine most interested in curcumin, (1E,6 E)-1,7-bis (4-hydroxy-3-methoxyphenyl)1,6-heptadien-3,5-dioni, which is extracted from Curcuma longa $L$. rhizomes and is widely utilized throughout Asia as a spice and herbal medicine (1). The neuroprotective effect of curcumin derived from its anti-inflammatory and antioxidant properties has been demonstrated in many studies $(7,10,21,23,24,29,30)$. Here, we investigated the effect of long-term dietary intake of curcumin by asking the following questions: Can curcumin be administered in food prior to ischemia? Does inclusion of curcumin to diet have a protective effect against stroke in the long term? Is there any difference in terms of superiority between administration before or after stroke? The majority of in vivo models of cerebral ischemia have relied on vessel occlusion predominantly affecting the forebrain (12). In this study, we aimed to evaluate the effects of long-term curcumin administration on neurological deficit scores, oxidative stress parameters, and apoptosis in the forebrain tissues of older rats subjected to experimental global ischemia and reperfusion.

\section{- MATERIAL and METHODS}

\section{Animals and Surgical Preparation}

All animal and experimental protocols were approved by the ethics committee of Bağcılar Training and Research Hospital (No. 2013-11). Forty 22-month-old female Wistar Hannover rats weighing 400-500 grams each were procured from the Experimental Research and Skill Development Center of Bağcılar Training and Research Hospital in this study. The maximum life expectancy of these rats is 26-28 months in our laboratory conditions. Therefore, 22-month-old rats, which had completed approximately $80 \%$ of their lifespan, were included. We confirmed that all animals were healthy before the experiments by assessing pre-surgery neurological scores.

During the study, the rats were kept in climate-controlled rooms at an ambient temperature of $21 \pm 2{ }^{\circ} \mathrm{C}$, with a humidity of $50-60 \%$ and a $12-\mathrm{h}$ light-dark cycle. Rats were kept in groups of eight in Type 4 cages produced from transparent polycarbonate. They had ad libitum access to standard rat feed and tap water. They were randomized into five groups of eight rats each, the details of which are provided below.

\section{Surgical Procedure for Global Ischemia Model}

After weighing the rats, they were anesthetized with intramuscular xylazine $10 \mathrm{mg} / \mathrm{kg}$ (Rompun, Bayer, Turkey) followed by intramuscular ketamine $65 \mathrm{mg} / \mathrm{kg}$ (Ketalar, Eczacıbaşı, Turkey). Subsequently, an appropriate area of skin was shaved and sterilized with $10 \%$ povidone iodine. After confirming a suitable level of anaesthesia based on a lack of response to a paw pinch test, the rat was placed in the supine position, covered with a sterile drape leaving access to the surgical site, and a midline neck incision was made under sterile conditions. Ischemic brain damage was induced in four groups (all groups except Group V), using the bilateral common carotid artery occlusion (BCCAO) method (25). Briefly, the skin layer was cut in a midline neck incision. Under microscopy, the muscular plane was reached by lifting the fatty tissues with a cranial pedicle flap, and the sternomastoid muscle was moved away with incisions from the cranial and caudal parts. The omohyoid muscle covering the carotid below the sternomastoid muscle was cut from the cranial part and removed from the carotid. The carotid and vagus nerve were cleared from the surrounding tissues and exposed. Both common carotid arteries were clamped for 30 
minutes, and reperfusion was carried out for 72 hours. In the ischemia group (Group IV), one rat died after 32 hours, which left a final $n$ of 7 .

\section{Drug Preparation and Treatment Schedule}

Curcumin (Sigma, St. Louis, MO, USA) was dissolved in 5\% alcohol, titrated to $\mathrm{pH} 7.4$ using $\mathrm{NaHCl}$, and diluted with physiological saline. Because it is considered a foodstuff, curcumin was given orally before ischemia, but it was given intraperitoneally after ischemia when the animals were unconscious and/or not eating normally.

There were three treatment groups. Group I received $300 \mathrm{mg} /$ $\mathrm{kg}$ oral curcumin every day for 21 days before ischemia and three times $300 \mathrm{mg} / \mathrm{kg}$ intraperitoneal curcumin treatments during the 72-hours reperfusion period after ischemia. Group II received three times $300 \mathrm{mg} / \mathrm{kg}$ intraperitoneal curcumin treatments during the 72-hours reperfusion period after ischemia. Group III received $300 \mathrm{mg} / \mathrm{kg}$ oral curcumin every day for 21 days before ischemia. Group IV underwent ischemia-reperfusion injury and did not receive curcumin. Group V animals underwent sham surgery; an incision was made, and they were injected with a matching volume of physiological saline.

\section{Neurological Deficits}

Neurological deficits were scored in all groups at 24 and 72 hours after ischemia induction by the same neurologist who was blinded to group allocation. The assessment was performed with a 5-point scale as described by Longa et al. (13). A score of 0 indicated no deficit; 1 , a failure to extend the right-left forepaw fully; 2 , circling to the right-left; 3 , falling to the right-left; 4 , no spontaneous walking and a depressed level of consciousness.

\section{Tissue Sample Extraction}

The rats were sacrificed under deep anaesthesia 72 hours after BCCAO. Cardiac formalin perfusion was performed before the rats were sacrificed to minimize brain tissue damage. The forebrain (including diencephalon and telencephalon) tissues were immediately extracted for assessment of biochemical parameters and histopathological examination. The samples analysed after overnight fixation were embedded in paraffin before 5- $\mu \mathrm{m}$ slices were prepared and stained with haematoxylin and eosin.

\section{Biochemical Parameters}

\section{Antioxidant Enzyme Activities and Lipid Peroxidation}

Xanthine dehydrogenase (XDH), superoxide dismutase (SOD), glutathione peroxidase (GPx), malondialdehyde (MDA) and catalase (CAT) levels were measured with the spectrophotometric method. SOD, CAT, and GPx enzyme activities, as well as and specification of the protein concentrations, were also determined. In brain homogenates, thiobarbituric acid was used to measure the level of MDA, which is the final product of lipid peroxidation.

\section{Cytokine Levels}

Concentrations of interleukin-6 (IL-6) and tumor necrosis factor-alpha (TNF-a) were determined using an enzyme-linked immunosorbent assay (ELISA) reader (BioTek Instruments, Winooksi, VT, USA) and immunometric ELISA kits (EIAab Wuhan Science Co. Ltd., Wuhan, China), according to the manufacturer's specifications. Results are expressed as pg/g of brain tissue.

\section{Determination of Fragmented Deoxyribonucleic Acid (DNA) in situ}

The apoptotic index was used for histopathological evaluation of the ischemic forebrain areas. Generally, it is defined as the percentage of apoptotic cells and bodies in the cell population. To determine the fraction of apoptotic cells, fractured DNA was labelled using terminal deoxynucleotidyl transferase (TdT)mediated nick end-labelling (TUNEL) in paraffin sections with an Apop Tag ${ }^{\circledR}$ peroxidase in situ apoptosis detection kit (EMD Millipore, Billerica, MA, USA). Sections were treated with 20 rg/ ml proteinase K (EC 3.4.21.64, Dako Inc., Glostrup, Denmark) for 10 minutes after deparaffinization and rehydration. Then, cells were treated with $3 \% \mathrm{H}_{2} \mathrm{O}_{2}$ in methanol for 5 minutes, TUNEL mixture for 1 hour (at $37^{\circ} \mathrm{C}$ ), and anti-digoxigeninperoxidase for 30 minutes.

Next, a 2\% diaminobenzidine solution was applied to the sections before they were counterstained with methyl green. The apoptotic index was detected in each section by microscopic evaluation. For this purpose, the numbers of TUNEL-positive cells and the total cell numbers were counted in five random areas under a $40 \times$ objective. The apoptotic index was calculated using the formula:

$\frac{\text { Mean number of TUNEL positive cells in five random fields }}{\text { Mean number of total cells in five random fields }} \times 100$

\section{Statistical Analysis}

The mean, standard deviation, median, and lowest and highest values were used in the descriptive statistics. The distributions of variables were verified with KolmogorovSmirnov tests. When the distribution was not normal, KruskalWallis and Mann-Whitney $U$ tests (for dual subgroup analyses) were used to analyse quantitative data. SPSS 22.0 (IBM Corp., Armonk, NY, USA) was used for the analyses. Differences were considered significant at $p<0.05$.

\section{RESULTS}

\section{Neurological Score}

Neurological evaluations were performed 24 and 72 hours after ischemia (Figure 1). While the 24-hour neurological score was $3.4 \pm 0.5$ on average in the ischemia group (Group IV), the neurological score was low in all treatment groups (Groups I, II, and III) compared to baseline. Treatment with curcumin (Groups I, II, and III) significantly decreased the post-ischemia neurological score by approximately $48 \%$ compared with Group IV $(p<0.05)$. Although the lowest score was obtained for Group III, no significant differences were observed in curcumin-treated animals $(p>0.05)$. Similarly, while the mean neurological score was $3.6 \pm 0.5$ in the ischemia group (Group IV) at 72 hours, the neurological score was lower in 
all treatment groups. The 72-hour neurological scores in the treatment groups (Groups I, II, and III) were significantly lower than in Group IV $(p<0.05)$. Treatment with curcumin (in Groups I, II, and III) significantly decreased the neurological score by approximately 54\% compared with Group IV. Although the lowest score was observed for Group III, there was no difference between the curcumin-treated groups ( $p>0.05)$. In the comparison of reperfusion at 24 and 72 hours, a slight increase was observed in neurological deficit score at 72 hours in the ischemia group (Group IV), while a slight reduction in the neurological score was noted in the two groups that received curcumin before ischemia (Groups I and III, Table I).

\section{Biochemical Parameters}

\section{Antioxidant Enzyme Activities and Lipid Peroxidation}

Concentrations of SOD, GPx, and CAT were significantly higher in curcumin-treated rats than those in Group IV $(p<0.05)$. Tissue concentrations of SOD, GPx, and CAT were significantly higher in Group I than in Groups II and III $(p<0.05)$. Concentrations of xanthine dehydrogenase and MDA values were significantly lower in Group I than in Groups II and III [( $p$ $<0.05$ ) (Figure 2)].

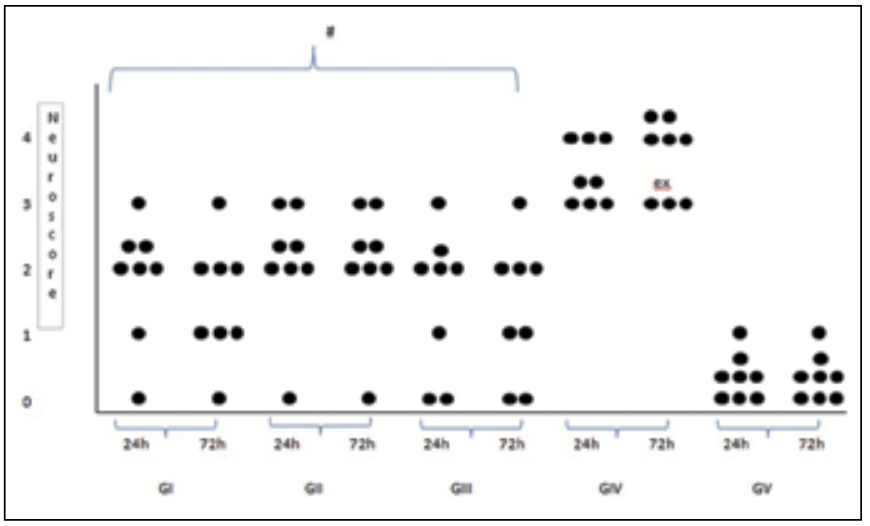

Figure 1: Neurologic deficit scores among the groups. A significant improvement in neurological score was noted in curcumin-treated rats. The lowest value was observed in Groups I and III (\# treatment groups).

\section{Cytokine Levels}

The concentration of IL-6 was significantly lower in the curcumin-treated groups than in Group IV $(p<0.05)$. The concentration of IL-6 was significantly lower in Group I compared to Groups II and III $(p<0.05)$. Similarly, TNF-a concentrations were significantly lower in the curcumin-treated groups than in Group IV $(p<0.05)$. The TNF-a concentration was significantly lower in Group I than in Groups II and III (p $<0.05$, Figure 2).

\section{Histopathological Changes}

While age-related degenerative changes were present in haematoxylin and eosin-stained forebrain sections in the sham group (Group V), marked areas of necrosis were only observed in the ischemia group. The lesions, which were mostly observed at the substantia alba level, had a spongious appearance with pyknotic cell nuclei in the background. Axonal structures had a sparse oedematous appearance with granulations, as well as occasional cell deletions. In the perineural gaps, cells contracted and became degenerative and necrotized in areas with ischemic damage in the substantia grisea, while granular and vacuolar changes were detected in neurophils. These alterations were more obvious in the ischemia group, while there was minimal cell damage in these areas in the treatment groups. Shrunken cytoplasm, atrophic neurons, and damaged nuclei were observed in the ischemia group (Figure 3A-J).

\section{Apoptotic Index}

The apoptotic index was highest in Group IV (Figure 4). A significant difference was noted $(p=0.000)$ when comparing this group with the treatment groups $(p<0.005)$. Treatment with curcumin (Group I) significantly decreased the apoptotic score by $50 \%$ compared with Group IV. The lowest apoptotic index was achieved in Group I, followed by Groups II and III. Although the lowest index score was in Group I, no significant difference was observed between the curcumin-treated groups $(p>0.05)$.

\section{DISCUSSION}

The results of this study demonstrate the neuroprotective effect of chronic curcumin supplementation in an ischemic

Table I: Neuro-Score and Apoptotic Index of the Ischemia Group and Curcumin Treated Rats

\begin{tabular}{|c|c|c|c|c|c|c|c|}
\hline & & Group I\# & Group II" & Group III & Group IV & Group V & $\mathbf{p}$ \\
\hline $72 \mathrm{~h}$ & mean $\pm S D$ & $1.4 \pm 1.1$ & $2.0 \pm 0.9$ & $1.5 \pm 0.9$ & $3.6 \pm 0.5$ & $0.1 \pm 0.4$ & 0.000 \\
\hline
\end{tabular}

"Treatment groups. CUR supplementation improved both the neurologic deficit score and apoptotic score. The results were significantly different from the corresponding sham operated group (as control) value at $p<0.005$. Kruskal-Wallis (Mann-Whitney $U$ test). Please note that the lowest score was in the Gl group. 
stroke model of older rats. We showed that antioxidant enzyme levels increased in animals that received curcumin before and after ischemia compared to those who received acute administration after ischemia. Furthermore, we also found that inflammatory cytokine levels were significantly lower in animals that received chronic curcumin administration compared to those that were only treated after ischemia. Notably, the lowest neurological and apoptotic scores were observed for Group I, which received curcumin administration before and after ischemia. These data provide evidence that curcumin could be a useful nutritional therapy in the future.
Ischemic stroke is the third most frequent cause of death in Europe and the United States $(6,8,26)$. Currently, the only approved medical treatment involves administration of intravenous recombinant tissue plasminogen activator within 4.5 hours after stroke onset to restore cerebral blood flow. Even if a patient survives an acute episode of cerebral ischemia, nearly 15-30\% of patients remain disabled after 3 months, and $20 \%$ require continuous medical treatment and supportive care (8). Therefore, novel, more effective therapies are urgently needed for stroke, which is a major public health issue. A series of mechanisms involved in ischemic
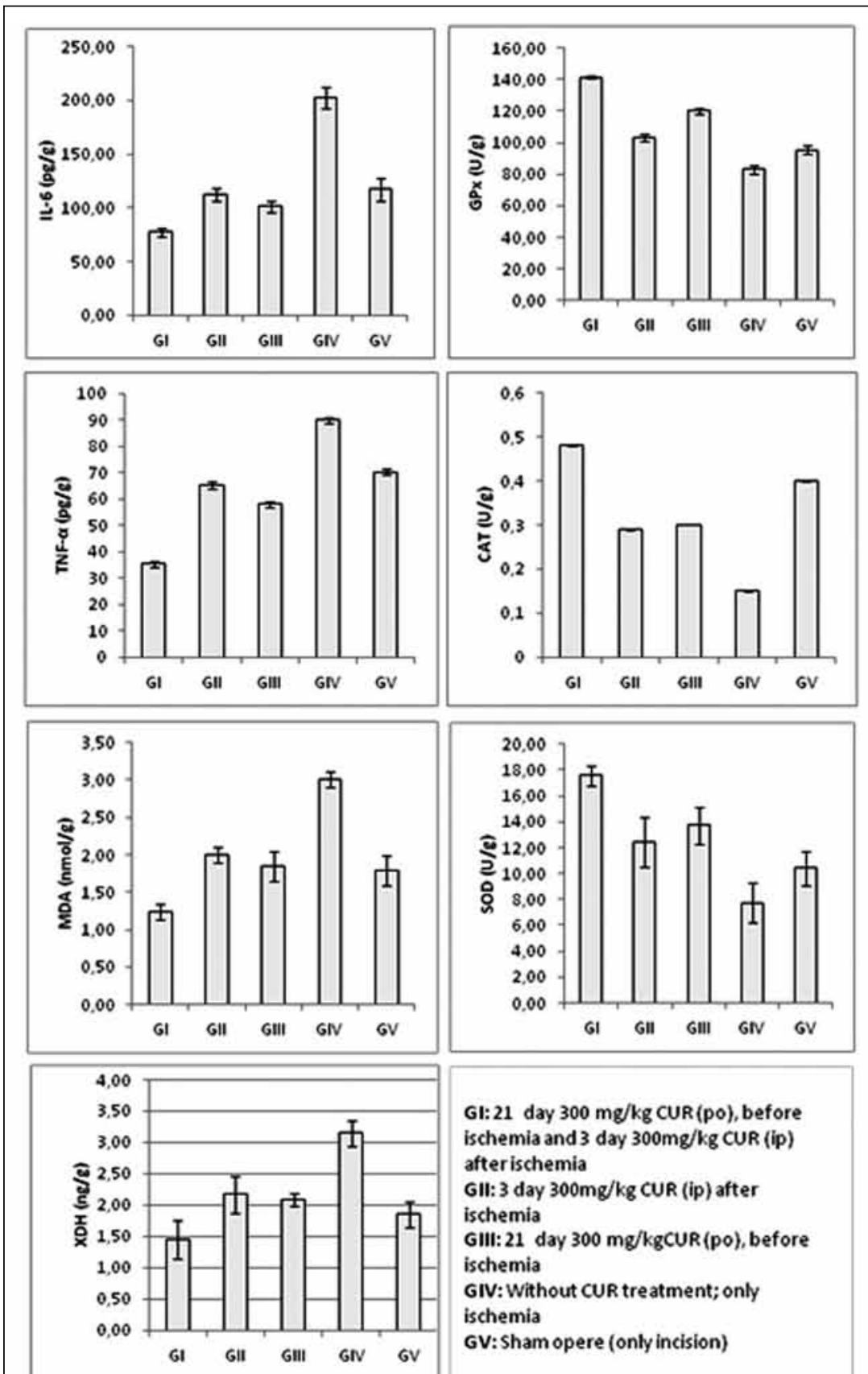

GI: 21 day $300 \mathrm{mg} / \mathrm{kg}$ CUR (po), before ischemia and 3 day $300 \mathrm{mg} / \mathrm{kg}$ CUR (ip) afterischemia

GII: 3 day $300 \mathrm{mg} / \mathrm{kg}$ CUR (ip) after ischemia

GIII: 21 day $300 \mathrm{mg} / \mathrm{kgCUR}$ (po), before ischemia

GIV: Without CUR treatment; only ischemia

GV: Sham opere (only incision)
Figure 2: Effect of curcumin on antioxidant enzyme activities and lipid peroxidation in rat forebrain tissue. All parameters were significantly different from the corresponding control (Group V) value $(p<0.05)$. 


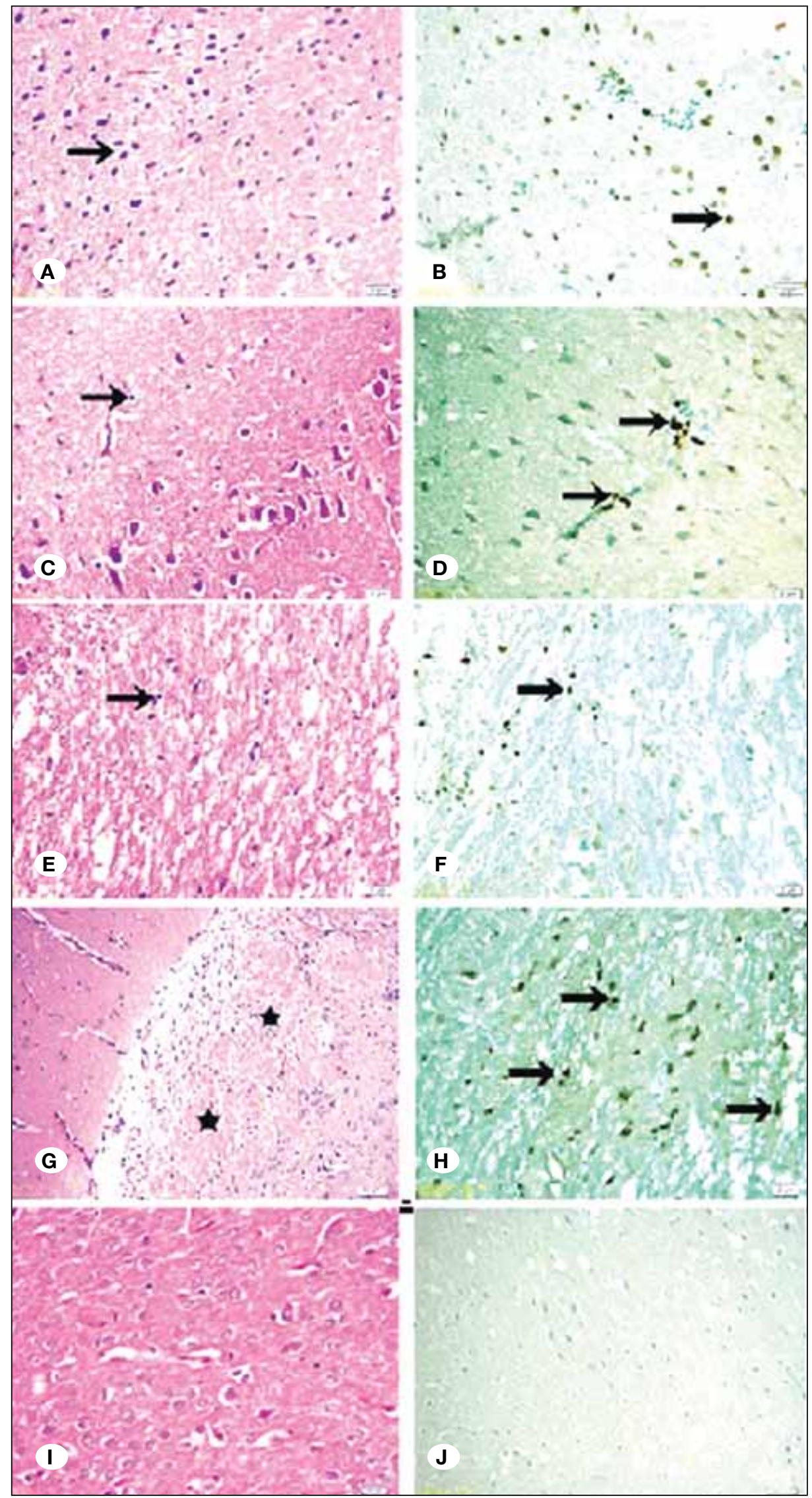

Figure 3: Haematoxylin and eosin staining (left column; Groups I to $V$ from top to bottom) revealed neuronal alterations in the brain after 72-hour reperfusion. Curcumin reduced secondary neuronal injury after reperfusion. Administration of curcumin $(300 \mathrm{mg} / \mathrm{kg}$ before and after ischemia-A-) ameliorated ischemiainduced neuronal damaged secondary to bilateral common carotid artery occlusion (BCCAO) more than $\mathrm{C}$ and $\mathrm{E}$. *, necrotic area in ischemia. (G) Please compare to curcumin treatment groups (A,C,E) and sham operated group (I) Terminal deoxynucleotidyltransferase (TdT)-mediated nick end-labelling (TUNEL) (right column) showing the effect of curcumin on DNA fragmentation after ischemiareperfusion. Fewer TUNEL-positive cells were observed in the curcumin groups, and they were thinly scattered in the penumbra. (B,D,F) Some TUNELpositive cells were located in the cortex of the curcumin-treated group (300 mg/ $\mathrm{kg}$, after ischemia). (D) There were fewer TUNEL-positive cells in the groups pretreated with curcumin than B and, $F$. No TUNEL-positive cells were found in the sham group. (J) Numerous TUNELpositive cells were found in the ischemic zone (H). 


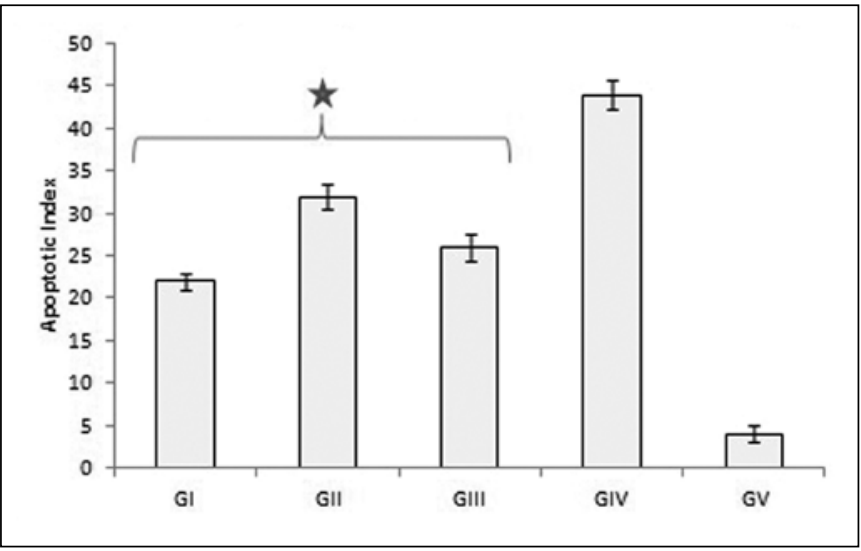

Figure 4: Apoptotic index in the ischemic area 72 hours after BCCAO. A significant decrease was noted in curcumin-treated groups. The lowest value is observed in Group I (*: treatment groups).

brain damage have been elucidated in previous studies and demonstrate that neuronal damage occurring after ischemia and reperfusion might activate apoptosis via oxidative stress or mitochondrial impairment $(12,14,17,20)$. Thus, antioxidant and anti-apoptotic agents are a focal point for the development of neuroprotective drugs in the treatment of stroke (9).

Our results show that curcumin preserves antioxidant enzymes such as superoxide dismutase, catalase, and glutathione peroxidase, and leads to a decrease in lipid peroxidation in rat forebrain tissue. Substances isolated from $C$. longa display strong antioxidant activities against a linoleic acid-rich environment in a model system and in vitro peroxidation of lipids found in the brain (18). Many rat cerebral ischemic model studies have demonstrated a significant neuroprotective effect of curcumin via its abilities to inhibit lipid peroxidation, increase antioxidant defence enzymes, and reduce peroxynitrite formation $(7,10,21,23,24,29,30)$.

In the present study, we investigated the effects of chronic administration of curcumin before ischemia on antioxidant levels, apoptosis, and neurological dysfunction in a global ischemia model in older rats. Our results showing a neuroprotective effect are in line with those of other studies $(7,10,21,23,24,29,30)$. Furthermore, we found that chronic curcumin administration before and after ischemia had more significant effects on antioxidant activities and cytokine levels than acute curcumin administration after ischemia.

Cerebral ischemia initiates molecular events triggered by the energy deficiency that occurs following reduced cerebral blood flow. The decrease in ATP leads to membrane depolarization and permeability dysfunction and causes increases in intracellular sodium, calcium, and chloride levels and extracellular potassium levels (3). Free radical formation is stimulated with activation of nitric oxide synthase, causing nitric oxide formation and intracellular calcium accumulation $(10,12)$. Toxic oxygen radicals are also produced in ischemic tissue. After reperfusion, free oxygen radicals and superoxide radicals cause endothelium damage and enhance vascular permeability. Moreover, activated adhesion molecules and cytokines initiate a systemic inflammatory response $(2,12,17)$. We measured biochemical parameters related to oxidative stress in the brain tissue at 72 hours after ischemia. We determined that levels of antioxidants including SOD, CAT, and GPX were higher in the treatment group than in the ischemia-only group. Similar findings have been published previously $(1,19)$. However, unlike earlier studies, we found that the antioxidant levels achieved with curcumin administration before and after ischemia were higher and more significant than acute administration after ischemia alone.

TNF- $a$ and IL- 6 are early mediators of inflammation and share the same signalling molecules as nuclear factor kappa-B and AP-1 transcription factor (15). Thus, both cytokines have similar biological effects. Curcumin reduces the production of proinflammatory cytokines such as TNF-a and IL-6. We also found significantly lower TNF-a and IL-6 levels in the curcumin group compared to the ischemia group $(p<0.05)$. Furthermore, we also detected that with curcumin administration before and after ischemia, cytokine levels were significantly lower than with acute administration after ischemia.

There are at least three recognizable pathways of ischemic cell death: necrosis, apoptosis, and, very probably, autophagocytosis. When evaluated under light microscopy, these cells are intensely acidophilic (eosinophilic), which is a defining characteristic of this morphological stage; they are also significantly argyrophilic (12). We focused on these cells in our histopathologic assessments.

The physiopathology of global ischemia is completely different from that of focal ischemia. Focal ischemia leads to a centre of infarcted tissue surrounded by a penumbral region that is not fully damaged. The blood flow is zero at the centre but varies in the surrounding region, and it is this region that constitutes the actual treatment target (12). In assessments of ischemic models in the literature, there are considerable differences in terms of duration and method. However, the important feature is whether any neurological deficit is clinically measureable after treatment. The fact that the average neurological deficit score in our ischemic group was 3.57 is clinical proof of a successful ischemic treatment model.

We observed that neurological deficit scores were significantly reduced at 24 and 72 hours in groups treated with curcumin after ischemia. Jiang et al. (10) demonstrated a significant reduction in neurological scores in rats that received $0.5-1$ and $2 \mathrm{mg} / \mathrm{kg}$ intravenous curcumin after ischemia. Yang et al. (28) described a significant decrease in neurological deficit by 24 hours with 50 and $100 \mathrm{mg} / \mathrm{kg}$ intraperitoneal curcumin treatment after ischemia. Similarly, Zhao et al. (30) found reduced neurological deficits at 24 hours with 100 and 300 $\mathrm{mg} / \mathrm{kg}$ intraperitoneal curcumin administration after ischemia. In line with our study, Zhao et al. (29) reported a meaningful improvement by both 24 and 72 hours; they obtained the best response with a $300 \mathrm{mg} / \mathrm{kg}$ dose (out of 100, 300 and $500 \mathrm{mg} / \mathrm{kg}$ intraperitoneal curcumin after ischemia). The only study in the literature that we could find in which curcumin was administered for a short period before and after ischemia was by Shukla et al. (21). They induced ischemia for 2 hours 
Table II: Examples of Previous Studies on CUR Administration Before Ischemia

\begin{tabular}{|c|c|c|c|}
\hline Author & Ischemia model & CUR before ischemia & CUR after ischemia \\
\hline \multirow[t]{2}{*}{ Shukla et al. (21) } & MCAO $2 \mathrm{~h}$ and & 5 days 100 mg/kg p.o. & 3 days 100 mg/kg p.o. \\
\hline & $72 \mathrm{~h}$ reperfusion & & \\
\hline Wu et al. (27) & No & *4 weeks 500 mg/kg p.o. & \\
\hline Present study & $72 \mathrm{~h}$ reperfusion & & \\
\hline
\end{tabular}

MCAO: Middle Cerebral Artery Occlusion, BCCAO: Bilateral Common Carotid Artery Occlusion. *Only CUR supplement without ischemia.

and applied 72-hour reperfusion; they administered $100 \mathrm{mg} /$ $\mathrm{kg}$ oral curcumin 5 days before ischemia and 3 days after ischemia. They reported that the neurological deficit had dropped markedly by 72 hours.

While neurological deficits were clearly evident in the ischemic group in our study, the histologic changes were less obvious. In fact, a study that confirmed our findings reported insignificant neuronal death despite reduced mitochondrial oxidative phosphorylation and microcirculation after global ischemia induced by 3-hour BCCAO occlusion (22). Fortunately, we evaluated cell death with TUNEL and detected apoptotic cells. In our global ischemic model induced by BCCAO, we were able to assess the whole brain and evaluate the effects of curcumin. The most important limitation of our study was that we did not measure the infarct volume.

A neuroprotective effect was also achieved in a few studies where curcumin was administered as a dietary supplement (Table II). Bala et al. (1) did not induce ischemia but published results showing antioxidative, anti-ageing, and neuroprotective effects of chronic curcumin administration at a $30 \mathrm{mg} / \mathrm{kg}$ oral dose given for 30 days. In a transient ischemic model induced with middle cerebral artery occlusion, Shukla et al. (21) orally administered curcumin at $100 \mathrm{mg} / \mathrm{kg}$ for 5 days before ischemia and detected a significant inhibition of lipid peroxidation and a significant increase in superoxide dismutase activity, leading them to conclude that curcumin had neuroprotective effects. Wu et al. (27) reported that oral administration of curcumin at a $550 \mathrm{ppm}$ dose for 30 days in a rat model of traumatic brain damage dramatically reduced oxidative damage.

The antioxidant activities of curcumin and its highly important bioactivities such as induction of cell apoptosis, inhibition of cell proliferation, anti-cell adhesion and motility, and anti-angiogenesis and anti-microbial activities have been investigated in vivo and in vitro. In the light of these findings, curcumin has been used in clinical trials to treat various inflammatory diseases and cancer (16). There is an increasing interest in curcumin in herbal medicine as a result of scientific studies demonstrating its beneficial pharmacological effects. Owing to its antioxidant and anti-inflammatory activities and perfect safety profile, curcumin may be a potential candidate in preventing or treating some diseases. It will be important to focus on the clinical administration of curcumin, which has shown no toxicity (5) even at doses of up to $8 \mathrm{~g}$ for 3 months and only had minor effects on the gastrointestinal system, to treat neurodegenerative diseases, diabetes, and cardiovascular diseases.

\section{- CONCLUSION}

Our study, which provides new data on biochemical parameters, neurological deficits, and apoptosis, differs from other studies and is more extensive because of our model system that investigated the long-term impact of high-dose curcumin treatment in older rats. The common aspect agreed on and scientifically demonstrated by researchers is that curcumin has antioxidant and neuroprotective effects. The present findings indicate that the long-term administration of curcumin at a high dose could be useful to prevent the negative effects of stroke; furthermore, these findings support that curcumin is a safe herbal agent that may be added to the diet. Considering its promising outcomes in clinical practice and its protective effect against ischemic stroke, we may soon see curcumin on pharmacy shelves.

\section{ACKNOWLEDGMENT}

This work was funded by the Ministry of Health, Health Science University, Bağcılar Training and Research Hospital, Research Funding (Grant No. 2013/11).

\section{REFERENCES}

1. Bala K, Tripathy BC, Sharma D: Neuroprotective and anti-ageing effects of curcumin in aged rat brain regions. Biogerontology 7: 81-89, 2006

2. Biswas SK, McClure D, Jimenez LA, Megson IL, Rahman I: Curcumin induces glutathione biosynthesis and inhibits NF-kappaß activation and interleukin-8 release in alveolar epithelial cells: Mechanism of free radical scavenging activity. Antioxid Redox Signal 7:32-41, 2005

3. Castellanos M, Serena J: Applicability of biomarkers in ischemic stroke. Cerebrovasc Dis 24 Suppl 1: 7-15, 2007

4. Eigner D, Scholz D: Ferula asa-foetida and Curcuma longa in traditional medical treatment and diet in Nepal. J Ethnopharmacol 67:1-6, 1999 
5. Fan X, Zhang C, Liu DB, Yan J, Liang HP: The clinical applications of curcumin: Current state and the future. Curr Pharm Des 19: 2011-2031, 2013

6. Feigin VL: Stroke epidemiology in the developing world. Lancet 365: 2160-2161, 2005

7. Ghoneim Al, Abdel-Naim AB, Khalifa AE, El-Denshary ES: Protective effects of curcumin against ischaemia/reperfusion insult in rat forebrain. Pharmacol Res 46:273-279, 2002

8. Go AS, Mozaffarian D, Roger VL, Benjamin EJ, Berry JD, Blaha MJ, Dai S, Ford ES, Fox CS, Franco S, Fullerton HJ, Gillespie C, Hailpern SM, Heit JA, Howard VJ, Huffman MD, Judd SE, Kissela BM, Kittner SJ, Lackland DT, Lichtman JH, Lisabeth LD, Mackey RH, Magid DJ, Marcus GM, Marelli A, Matchar DB, McGuire DK, Mohler ER 3rd, Moy CS, Mussolino ME, Neumar RW, Nichol G, Pandey DK, Paynter NP, Reeves MJ, Sorlie PD, Stein J, Towfighi A, Turan TN, Virani SS, Wong ND, Woo D, Turner MB; American Heart Association Statistics Committee and Stroke Statistics Subcommittee: Heart disease and stroke statistics-2014 update: A report from the American Heart Association. Circulation 129: 399-410, 2014

9. Han Z, Xiao MJ, Shao B, Zheng RY, Yang GY, Jin K: Attenuation of ischemia-induced rat brain injury by 2-(-2-benzofuranyl)2-imidazoline, a high selectivity ligand for imidazoline I(2) receptors. Neurol Res 31:390-395, 2009

10. Jiang J, Wang W, Sun YJ, Hu M, Li F, Zhu DY: Neuroprotective effect of curcumin on focal cerebral ischemic rats by preventing blood-brain barrier damage. Eur J Pharmacol 561: 54-62, 2007

11. Lindgren A: Stroke genetics: A review and update. J Stroke 16: 114-123, 2014

12. Lipton P: Ischemic cell death in brain neurons. Physiol Rev 79: 1431-568, 1999

13. Longa EZ, Weinstein PR, Carlson S, Cummins R: Reversible middle cerebral artery occlusion without craniectomy in rats. Stroke 20: 84-91, 1989

14. Mills JC: Mechanisms underlying the hallmark features of the execution-phase of apoptosis. In: Mattson MP, Estus S, Rangenakar VM (eds). Programmed Cell Death. Vol: 1, Amsterdam: Elsevier Press, 2001: 1-38

15. Nguyen MD, Julien JP, Rivest S: Innate immunity: The missing link in neuroprotection and neurodegeneration. Nat Rev Neurosci 3: 216-227, 2002

16. Noorafshan A, Ashkani-Esfahani S: A review of therapeutic effects of curcumin. Curr Pharm Des 19: 2032-2046, 2013

17. Schaller B, Graf R: Cerebral ischemia and reperfusion: The pathophysiologic concept as a basis for clinical therapy. $\mathrm{J}$ Cereb Blood Flow Metab 24: 351-371, 2004
18. Sharma OP: Antioxidant activity of curcumin and related compounds. Biochem Pharmacol 25: 1811-1812, 1976

19. Sharma RA, Steward WP, Gescher AJ: Pharmacokinetics and pharmacodynamics of curcumin. Adv Exp Med Biol 595: 453470, 2007

20. Shi S, Yang W, Tu X, Chen C, Wang C: Ischemic preconditioning reduces ischemic brain injury by suppressing nuclear factor kappa B expression and neuronal apoptosis. Neural Regen Res 8: 633-638, 2013

21. Shukla PK, Khanna VK, Ali MM, Khan MY, Srimal RC: Antiischemic effect of curcumin in rat brain. Neurochem Res 33: 1036-1043, 2008

22. Suchadolskiene O, Pranskunas A, Baliutyte G, Veikutis V, Dambrauskas Z, Vaitkaitis D, Borutaite V: Microcirculatory, mitochondrial, and histological changes following cerebral ischemia in swine. BMC Neurosci 15: 2-9, 2014

23. Thiyagarajan $M$, Sharma SS: Neuroprotective effect of curcumin in middle cerebral artery occlusion induced focal cerebral ischemia in rats. Life Sci 74: 969-985, 2004

24. Wang $Q$, Sun $A Y$, Simonyi A, Jensen MD, Shelat $P B$, Rottinghaus GE, MacDonald RS, Miller DK, Lubahn DE, Weisman GA, Sun GY: Neuroprotective mechanisms of curcumin against cerebral ischemia-induced neuronal apoptosis and behavioral deficits. J Neurosci Res 82: 138-148, 2005

25. Wang Q, Xu J, Rottinghaus GE, Simonyi A, Lubahn D, Sun GY, Sun AY: Resveratrol protects against global cerebral ischemic injury in gerbils. Brain Res 958: 439-447, 2002

26. WHO: Neurological disorders; a public health approach. In: Neurological Disorders Public Health Challenges. Switzerland: WHO Press, 2006:151-163

27. Wu A, Ying Z, Gomez-Pinilla F: Dietary curcumin counteracts the outcome of traumatic brain injury on oxidative stress, synaptic plasticity, and cognition. Exp Neurol 197: 309-317, 2006

28. Yang C, Zhang X, Fan H, Liu Y: Curcumin upregulates transcription factor Nrf2, $\mathrm{HO}-1$ expression and protects rat brains against focal ischemia. Brain Res 1282: 133-141, 2009

29. Zhao J, Yu S, Zheng W, Feng G, Luo G, Wang L, Zhao Y: Curcumin improves outcomes and attenuates focal cerebral ischemic injury via antiapoptotic mechanisms in rats. Neurochem Res 35: 374-379, 2010

30. Zhao J, Zhao Y, Zheng W, Lu Y, Feng G, Yu S: Neuroprotective effect of curcumin on transient focal cerebral ischemia in rats. Brain Res 1229: 224-232, 2008 